\title{
Impact of propofol on renal ischemia/reperfusion endoplasmic reticulum stress ${ }^{1}$
}

\author{
Mengqin Su', Sueng Ren', Wei Zhong', Xueping Han"
}

'PhD, Department of Anesthesiology, Henan Provincial Chest Hospital, Zhengzhou, China. Conception and design of the study, analysis and interpretation of data, manuscript writing, critical revision.

"Professor, Department of Anesthesiology, First Affiliated Hospital, Zhengzhou University; Institute of Clinical Medical Research, Henan Universities, Zhengzhou, China. Conception, design and intellectual content of the study, supervised all phases of the study.

\begin{abstract}
Purpose: To investigate the protective mechanisms of propofol (Pro) on renal ischemia/reperfusion (I/R) injury by studying its impact on renal I/R endoplasmic reticulum stress.

Methods: Eighteen male Sprague-Dawley rats (SD rats) were randomly divided into three groups: the I/R group, the Pro pretreatment group, and the control group, and corresponding treatments were performed. The levels of serum creatinine $(\mathrm{Cr})$ and blood urea nitrogen (BUN) of each group were detected. The expression levels of CCAAT-enhancer-binding protein (C/EBP) homology protein (CHOP) and caspase-12 protein within renal tissue samples were detected by western blot.

Results: The periodic acid-Schiff (PAS) staining was performed to observe the morphological changes within the renal tissues, and the terminal deoxynucleotidyl transferase-mediated dUTP-biotin nick end labeling (TUNEL) assay was performed to detect the presence of renal apoptosis. The Pro pretreatment significantly reduced the serum $\mathrm{Cr}$ and BUN levels, as well as the expressions levels of CHOP and caspase-12 protein inside the kidney of I/R rats, improving renal pathological injury and reducing the I/R-induced renal apoptosis.

Conclusion: Propofol could downregulate the expression of stress-apoptotic proteins CHOP and caspase-12 in the endoplasmic reticulum, thus reducing renal I/R injury.

Key words: Endoplasmic Reticulum Stress. Reperfusion Injury. Propofol. Kidney. Rats.
\end{abstract}




\section{Introduction}

Renal ischemia/reperfusion (I/R) injury is the main cause of acute renal failure. It is common in clinical patients with hypovolemic shock, kidney transplantation, cardiovascular surgery, etc., and can be life threatening ${ }^{1}$. Renal $\mathrm{I} / \mathrm{R}$ is known to promote the upregulation of CCAAT-enhancer-binding protein (C/EBP) homology protein (CHOP), therefore increasing apoptosis $^{2}$. CHOP and caspase-12 are key proteins in the endoplasmic reticulum stress apoptosis pathway. Hence, it is particularly important to have measures that can promptly prevent this type of injury. Studies have found that intravenous anesthetic propofol (Pro) shows properties such as antioxidation, anti-

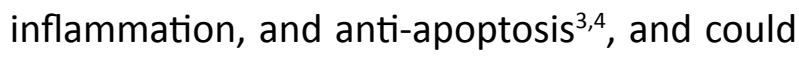
reduce $I / R$ injury in organs including the liver, kidney, and brain ${ }^{5-7}$; however, its effects on the signals of the endoplasmic reticulum stress apoptosis pathway, during renal I/R injury, are yet to be reported. In this study, we observed the effects of Pro on the expressions of $\mathrm{CHOP}$ and caspase-12 in renal I/R injury, aiming to explore the molecular mechanisms related to the actions of Pro in renal I/R injury prevention.

\section{Methods}

The animal use protocol has been reviewed and approved by the Institutional Animal Care and Use Committee (IACUC) of Zhengzhou University. This study was carried out in strict accordance with the recommendations in the Guide for the Care and Use of Laboratory Animals of the National Institutes of Health.

The male SD rats (SPF grade, purchased from the Experimental Animal Center of the Henan Province), weighed 250-300 g, and had unrestricted access to food and water. All the animal-related operations were in line with the regulations of the experimental animal management.

\section{Preparation of the renal $I / R$ model and animal groupings}

To produce the renal I/R injury model in rats, anesthesia was induced by intraperitoneal injection of $60 \mathrm{mg} / \mathrm{kg}$ sodium pentobarbital. Then the rat was placed on an operation plate equipped with a thermostat system. The rat's rectal temperature was maintained at $37^{\circ} \mathrm{C}$. The abdominal cavity was opened along the ventral abdominal midline and the right kidney was resected. The left renal pedicle was freed and an intraperitoneal injection of $50 \mathrm{U} / \mathrm{kg}$ heparin was administered; after $5 \mathrm{~min}$, the left renal pedicle was occluded with a noninjury artery clamp. The renal pedicle was reopened $60 \mathrm{~min}$ later. The abdominal cavity was then closed and after $24 \mathrm{~h}$, perfusion was performed and the blood serum and renal specimens were collected. The 18 male SD rats were randomly divided into three groups. The I/R group comprised rats that had undergone the procedure described above. The Pro pretreatment group comprised rats in which $25 \mathrm{mg} / \mathrm{kg} / \mathrm{h}$ Pro (Libang Pharmaceutical Co., Ltd., Xi'an, China) was pumped via the tail vein for two consecutive hours after anesthesia, and the renal I/R injury model was prepared during this time. Finally, the control group comprised rats that had been treated in the same manner as the I/R group except occlusion of the left renal artery pedicle. The serum and renal specimens of each group were sampled.

\section{Detection of serological indicators}

The blood was sampled from the inferior vena cava, and serum creatinine $(\mathrm{Cr})$ and blood urea nitrogen (BUN) were detected using a standard clinical automatic biochemical analyzer (Hitachi7600-020). 
Detection of renal CHOP and caspase-12 protein

We took $50 \mathrm{mg}$ of frozen renal tissue, extracted the proteins according to the instructions provided with the RIPA clearage solution and used a BCA protein concentration detection kit to detect the protein concentration (Beyotime Biotechnology Co. Ltd, Shanghai, China). Then, SDS sample-loading buffer was added to the isolated protein, and the mixture was boiled for $5 \mathrm{~min}$. A 10\% SDSpolyacrylamide separation gel was prepared, to which $20 \mu \mathrm{g}$ of protein was added per lane. Following electrophoresis and membrane transferring, 5\% nonfat milk was used to block the PVDF membrane. The primary CHOP antibody (Santa Cruz Biotech., California, USA) and primary caspase-12 antibody (Santa Cruz Biotech., California, USA) (1:1000) were added and the membrane was incubated overnight at $4^{\circ} \mathrm{C}$. The membrane was washed with TBST, and then, the appropriate secondary antibody (Beijing Kangwei Century Biotech. Co., Beijing, China) (1:5000) was added and the membrane was incubated for $1 \mathrm{~h}$ at room temperature. Following another wash with TBST, ECL luminescent was added to the membrane. After $5 \mathrm{~min}$, the blots were developed by exposure in darkness. The expression of $\beta$-actin protein (antibody purchased from Santa Cruz Biotech., California, USA) was used as a control to correct the calculated expression levels of the target proteins.

\section{Pathomorphological observations}

We cut the paraffin-embedded renal tissue samples into 5 - $\mu \mathrm{m}$-thick paraffin slices, followed by dewaxing, hydration, periodic acidSchiff (PAS) staining, hematoxylin re-staining, routine dehydration, hyalinization, and fixation of the samples. The pathomorphological changes of the renal tissues from each group were then observed under a microscope.

\section{Determination of renal cell apoptosis}

The tissue samples were cut into 5 - $\mu \mathrm{m}$-thick paraffin slices, dewaxed, and hydrated. The samples were then labeled according to the instructions of the terminal deoxynucleotidyl transferase-mediated dUTPbiotin nick end labeling (TUNEL) apoptosis kit (Chemicon International Co., California, USA). The apoptotic nuclei appeared brown. Each slice was observed in at least three nonoverlapping fields to count the number of the apoptotic cells.

\section{Statistical analysis}

SPSS17.0 statistical software was used for the statistical analysis; the quantitative data were analyzed by using a t-test, with $\mathrm{P}<0.05$ considered as a statistically significant difference.

\section{Results}

\section{Changes in serum Crand BUN}

The serum Cr levels of the I/R group, the Pro pretreatment group, and the control group were $201.3 \pm 27.5 \mu \mathrm{mol} / \mathrm{L}, 140.7 \pm 25.6 \mu \mathrm{mol} / \mathrm{L}$, and $37.7 \pm 9.3 \mu \mathrm{mol} / \mathrm{L}$, respectively. The serum BUN levels of the above three groups were $22.3 \pm 4.5 \mathrm{mmol} / \mathrm{L}, 11.6 \pm 5.3 \mathrm{mmol} / \mathrm{L}$, and 4.4 $\pm 3.3 \mathrm{mmol} / \mathrm{L}$, respectively. Compared with the I/R group, the Pro pretreatment group showed notably reduced serum $\mathrm{Cr}$ and BUN levels, and the differences were statistically significant $(P<$ 0.05).

\section{Expressions of $\mathrm{CHOP}$ and caspase-12 protein}

The expression levels of $\mathrm{CHOP}$ and caspase-12 protein in the I/R group significantly increased, while the Pro pretreatment significantly reduced the protein levels of $\mathrm{CHOP}$ and caspase-12 (Figure 1). 


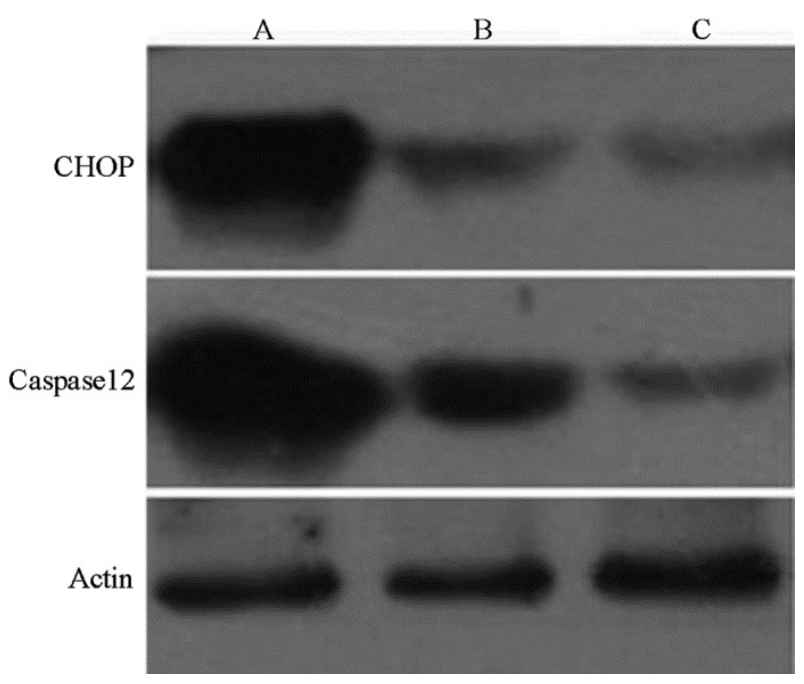

Figure 1 - Expressions of CHOP and Caspase12 protein in each group by Western blot. A: I/R group; B: Pro pretreatment group; C: Sham group.

\section{Renal pathomorphological changes}

The I/R group exhibited widespread necrosis of tubular epithelial cells, with a large number of tubular and necrotic cells found inside the tubular cavity. In this group, the renal interstitium showed vascular dilatation and congestion, with focal hemorrhage and inflammatory cell infiltration. The Pro pretreatment group only showed focal tubular necrosis; there were fewer tubular and necrotic cells, interstitial congestion and edema significantly reduced, and inflammatory cell infiltration was lessened. The control group showed normal renal tissue structures (Figure 2).

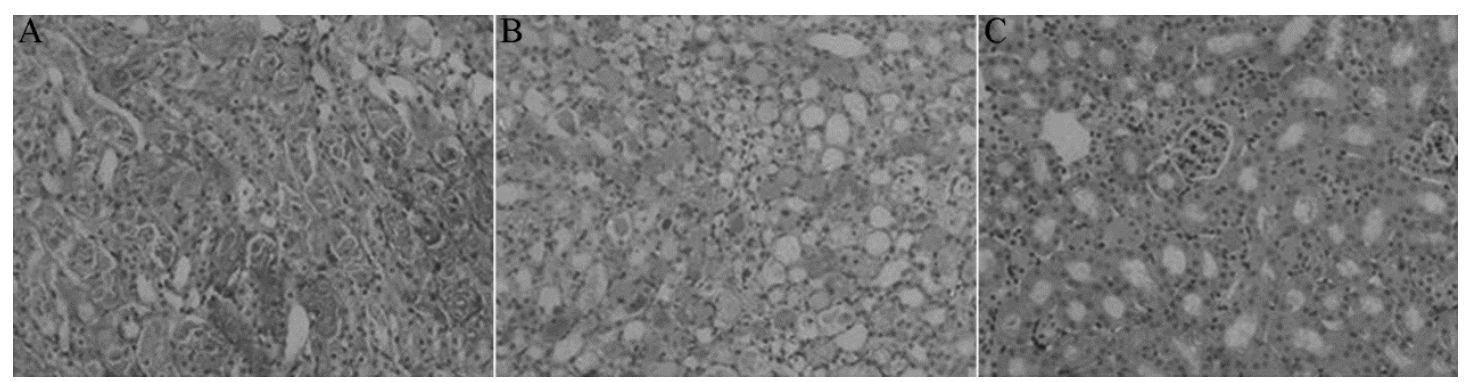

Figure 2 - Renal pathomorphological changes in each group (PAS, $\times 400)$. A: I/R group; B: Pro pretreatment group; C: Sham group.

\section{Renal cell apoptosis}

The number of apoptotic cells in the I/R group, the Pro pretreatment group, and the control group was $171 \pm 36,80 \pm 29$, and $5 \pm 3$ cells respectively per field of vision. Compared with the I/R group, the Pro pretreatment group showed significantly reduced number of apoptotic cells $(\mathrm{P}<0.05)$ (Figure 3$)$.

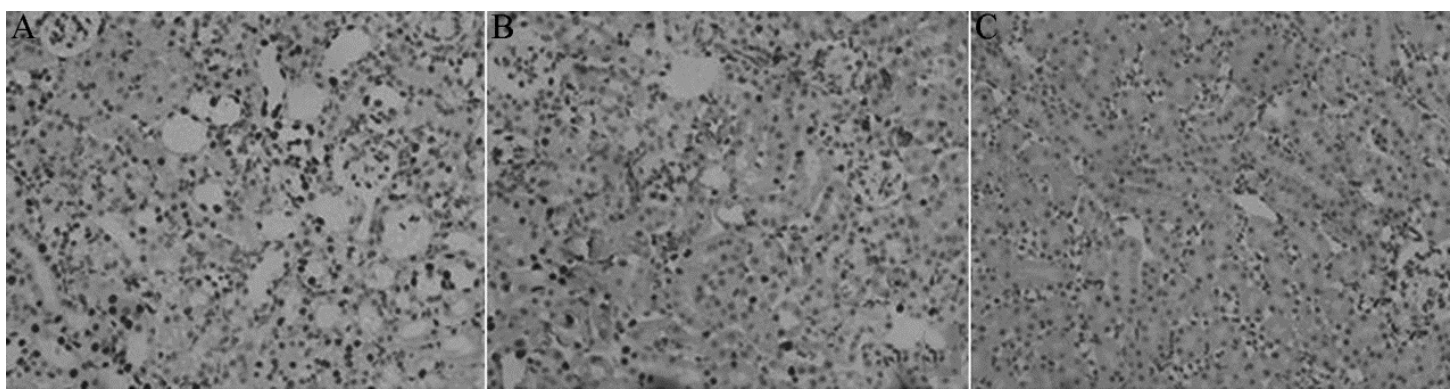

Figure 3 - Renal cell apoptosis in each group (TUNEL, ×400). A: I/R group; B: Pro pretreatment group; C: Sham group. 


\section{- Discussion}

Renal acute $I / R$ injury is the main cause of clinical acute renal failure, and its mortality rate is high ${ }^{8}$. The pathophysiological mechanism of I/R injury involves the generation of a large amount of free radicals, $\mathrm{Ca}^{2+}$ overload, and inflammatory cell infiltration?. When ischemia occurs, ATP is rapidly depleted, consequently the mitochondrial endoplasmic reticulum swells, and the change in membrane permeability causes the release of $\mathrm{Ca}^{2+}$ from the mitochondria and endoplasmic reticulum. During the period of reperfusion, the ATP pump has yet to be restored, so a large number of extracellular $\mathrm{Ca}^{2+}$ flows in, resulting in the rapid increase in intracellular $\mathrm{Ca}^{2+}$. The overload of $\mathrm{Ca}^{2+}$ impedes oxidative phosphorylation within the mitochondria, hinders ATP synthesis, and promotes the destruction of proteins and membrane structures. Consequently, a large number of oxygen free radicals are generated, and the cells exhibit apoptosis and even necrosis ${ }^{10}$. The kidney is rich in blood supply, so it is much more sensitive to ischemia; I/R causes an overload in renal $\mathrm{Ca}^{2+}$. Meanwhile, the rate of renal cell apoptosis increases, suggesting that $\mathrm{Ca}^{2+}$ overload is an important cause of renal dysfunction ${ }^{11}$. Certain interventions could be implemented at the initial stages of apoptosis to reverse it, which could reduce injury to some extent.

The endoplasmic reticulum stress apoptosis pathway is one of the important endogenous apoptosis pathways, and $\mathrm{CHOP}$ and caspase-12 are important apoptotic proteins involved in this pathway ${ }^{12}$. Endoplasmic reticulum stress is the progress indicator of renal injury ${ }^{13}$. I/R causes CHOP upregulation in the renal endothelial and epithelial cells, with an increase in apoptosis. Knocking out the CHOP gene was shown to significantly reduce renal $I / R$ injury in mice, and improve renal microcirculation after $I / R^{2}$. Furthermore, post-ischemic preconditioning was shown to reduce the I/R-induced renal endoplasmic reticulum stress by downregulating the protein expression of glucose regulating protein 78 (GRP78), activating transcriptional factor 4 (ATF4), double-stranded RNA-dependent protein kinase-like ER kinase (PERK) protein, $X$ box binding protein 1 (XBP-1), and caspase-12, thus alleviating renal $I / R$ injury ${ }^{14}$. Therefore, if endoplasmic reticulum stress could be subdued, renal $I / R$ injury could in turn be reduced. Certain studies found that Pro might regulate the unfolded protein response during endoplasmic reticulum stress, as to reduce apoptotic injury in neurons ${ }^{15}$. Another study found that Pro could reduce endoplasmic reticulum stress-mediated apoptosis in the neuroblastoma cell line, $\mathrm{SH}-\mathrm{SY}_{5} \mathrm{Y}^{16}$. The present study demonstrated that Pro reduces the expression levels of CHOP and caspase-12 protein in kidneys that have undergone I/R, as well as reduce renal cell apoptosis, thus reducing renal $I / R$ injury.

Pro is an intravenous anesthetic and sedative commonly used in clinical practice; it has a phenolic hydroxyl structure similar to that of vitamin $E$ and butylhydroxytoluene, and can act as a hydrogen donor in vivo, reacting with free radicals and generating phenoxy. Studies found that Pro could reduce LPSinduced macrophage inflammatory responses and reduce the generation of tumor necrosis factor $\alpha$ (TNF- $\alpha)$, interleukin (IL)-6, and IL10 by inhibiting the reactive oxygen species/ Akt/inhibitor kappa $B$ kinase $\beta /$ nuclear factor $\mathrm{kB}$ (ROS/Akt/IKK $\beta / N F-k B)$ signaling pathway ${ }^{17}$. Pro has been shown to play an anti-inflammatory role in cardiopulmonary bypass, reducing the production of IL- $6^{18}$, and to exert antioxidant effects in the $\mathrm{H} 9 \mathrm{c} 2$ cells via FoxO1, thus protecting myocardial cells ${ }^{19}$. Pro was reported to reduce renal I/R injury in rats with hyperglycemia, increase the level of superoxide dismutase, and reduce the levels 
of myeloperoxidase, IL-1 $\beta$, and TNF- $\alpha^{6}$. Pro can reduce post-I/R liver cell apoptosis by inhibiting the activation of glycogen synthase kinase $3 \beta$ (GSK-3 $\beta$ ) in mitochondria, and maintaining the opening of mitochondrial permeabilitytransition pores, thus preventing mitochondrial swelling and potential membrane downfall ${ }^{5}$. Furthermore, Pro was reported to also activate the Nrf2 pathway to alleviate liver oxidative stress ${ }^{20}$, while in human alveolar epithelial cells, Pro reduced LPS-induced ROS production, activated Nrf2/GSH and inhibited nicotinamide adenine dinucleotide phosphate (NADPH) oxidas $^{\mathrm{e} 3}$. Through inhibiting the NADPH oxidasemediated mastocyte activation, Pro is thought to alleviate intestinal I/R injury ${ }^{21}$. Ultimately, Pro has anti-apoptosis, anti-inflammation, and antioxidation properties.

\section{Conclusions}

Propofol was shown to inhibit the expression of key apoptotic proteins in the endoplasmic reticulum apoptotic signaling pathway, thus providing an anti-apoptotic protective effect against renal I/R injury. This broadens the theoretical foundation for the Pro-mediated protection of renal functions. Nonetheless, research related to the protective effects of Pro against renal I/R injury still requires further investigation.

\section{References}

1. Gueler F, Gwinner W, Schwarz A, Haller $\mathrm{H}$. Long-term effects of acute ischemia and reperfusion injury. Kidney Int. 2004 Aug;66(2):523-7. doi: 10.1111/j.15231755.2004.761_11.x.

2. Dong B, Zhou H, Han C, Yao J, Xu L, Zhang M, Fu Y, Xia Q. Ischemia/reperfusion-induced CHOP expression promotes apoptosis and impairs renal function recovery: the role of acidosis and GPR4. PLoS One. 2014 Oct 24;9(10)e110944. doi: 10.1371/journal. pone.0110944.
3. Hsu HT, Tseng YT, Hsu YY, Cheng KI, Chou SH, Lo YC. Propofol attenuates lipopolysaccharide-induced reactive oxygen species production through activation of $\mathrm{Nrf2} / \mathrm{GSH}$ and suppression of NADPH oxidase in human alveolar epithelial cells. Inflammation. 2015 Feb;38(1):415-23. doi: 10.1007/s10753-014-0046-4.

4. Tian Y, Guo S, Guo Y, Jian L. Anesthetic Propofol Attenuates Apoptosis, Abeta Accumulation, and Inflammation Induced by Sevoflurane Through NF-kappaB Pathway in Human Neuroglioma Cells. Cell Mol Neurobiol. 2015 Aug;35(6):891-8. doi: 10.1007/s10571-015-0184-8.

5. Zhao G, Ma $H$, Shen $X, X u G F$, Zhu $Y L$, Chen B, Tie R, Qu P, Lv Y, Zhang H, Yu J. Role of glycogen synthase kinase 3beta in protective effect of propofol against hepatic ischemia-reperfusion injury. J Surg Res. 2013 Nov;185(1):388-98. doi: 10.1016/j. jss.2013.05.004.

6. Yoo YC, Yoo KJ, Lim BJ, Jun JH, Shim JK, Kwak YL. Propofol attenuates renal ischemia-reperfusion injury aggravated by hyperglycemia. J Surg Res. 2013 Aug;183(2):783-91. doi: 10.1016/j. jss.2013.02.017.

7. Shi SS, Yang WZ, Chen Y, Chen JP, Tu XK. Propofol reduces inflammatory reaction and ischemic brain damage in cerebral ischemia in rats. Neurochem Res. 2014 May;39(5):793-9. doi: 10.1007/s11064-0141272-8.

8. Malek M, Nematbakhsh M. Renal ischemia/ reperfusion injury; from pathophysiology to treatment. J Renal Inj Prev. 2015 Jun 1;4(2):20-7. doi: 10.12861/jrip.2015.06.

9. Gracia-Sancho J, Casillas-Ramirez A, Peralta C. Molecular pathways in protecting the liver from ischaemia/reperfusion injury: a 2015 update. Clin Sci (Lond). 2015 Aug;129(4):34562. doi: 10.1042/CS20150223.

10.Garcia-Dorado D, Ruiz-Meana M, Inserte J, Rodriguez-Sinovas A, Piper HM. Calciummediated cell death during myocardial reperfusion. Cardiovasc Res. 2012 May 1;94(2):168-80. doi: 10.1093/cvr/cvs116.

11. Chatauret N, Badet L, Barrou B, Hauet T. Ischemia-reperfusion: From cell biology to acute kidney injury. Prog Urol. 2014 Jun;24 Suppl 1:S4-12. doi: 10.1016/S11667087(14)70057-0. 
12.Sano R, Reed JC. ER stress-induced cell death mechanisms. Biochim Biophys Acta. 2013 Dec;1833(12):3460-70. doi: 10.1016/j. bbamcr.2013.06.028.

13.Inagi R. Endoplasmic reticulum stress as a progression factor for kidney injury. Curr Opin Pharmacol. 2010 Apr;10(2):156-65. doi: 10.1016/j.coph.2009.11.006.

14.Mahfoudh-Boussaid A, Zaouali MA, Hauet T, Hadj-Ayed K, Miled AH, Ghoul-Mazgar S, Saidane-Mosbahi D, Rosello-Catafau J, Ben Abdennebi $\mathrm{H}$. Attenuation of endoplasmic reticulum stress and mitochondrial injury in kidney with ischemic postconditioning application and trimetazidine treatment. J Biomed Sci. 2012 Aug;19:71. doi: 10.1186/1423-0127-19-71.

15.Wang L, Tang $W$, Jiang $T$, Lu $P$, Li $Y$, Sun A, Shen $Y$, Chen $Y$, Wang $H$, Zong $Z$, Wang $Y$, Chen $L$, Shen $Y$. Endoplasmic reticulum stress is involved in the neuroprotective effect of propofol. Neurochem Res. 2014 Sep;39(9):1741-52. doi: 10.1007/s11064014-1369-0.

16.Nakajima A, Tsuji M, Inagaki M, Tamura Y, Kato M, Niiya A, Usui Y, Oguchi K. Neuroprotective effects of propofol on ER stress-mediated apoptosis in neuroblastoma SH-SY5Y cells. Eur J Pharmacol. 2014 Feb 15;725:47-54. doi: 10.1016/j.ejphar.2014.01.003.

17.Hsing CH, Lin MC, Choi PC, Huang WC, Kai JI,
Tsai CC, Cheng YL, Hsieh CY, Wang CY, Chang $\mathrm{YP}$, Chen $\mathrm{YH}$, Chen $\mathrm{CL}$, Lin CF. Anesthetic propofol reduces endotoxic inflammation by inhibiting reactive oxygen species-regulated Akt/IKKbeta/NF-kappaB signaling. PLoS One. 2011 Mar;6(3):e17598. doi: 10.1371/ journal.pone.0017598.

18.Samir A, Gandreti N, Madhere M, Khan A, Brown M, Loomba V. Anti-inflammatory effects of propofol during cardiopulmonary bypass: a pilot study. Ann Card Anaesth. 2015 Oct;18(4):495-501. doi: 10.4103/09719784.166451.

19.Zhao D, Li Q, Huang Q, Li X, Yin M, Wang Z, Hong J. Cardioprotective effect of propofol against oxygen glucose deprivation and reperfusion injury in $\mathrm{H} 9 \mathrm{c} 2$ cells. Oxid Med Cell Longev. 2015;2015:184938. doi: $10.1155 / 2015 / 184938$.

20.Ge M, Yao W, Wang Y, Yuan D, Chi X, Luo G, Hei Z. Propofol alleviates liver oxidative stress via activating Nrf2 pathway. J Surg Res. 2015 Jun 15;196(2):373-81. doi: 10.1016/j. jss.2015.03.016.

21.Gan X, Xing D, Su G, Li S, Luo C, Irwin MG, Xia Z, Li H, Hei Z. Propofol attenuates small intestinal ischemia reperfusion injury through inhibiting NADPH oxidase mediated mast cell activation. Oxid Med Cell Longev. 2015;2015:167014. doi: $10.1155 / 2015 / 167014$.

\section{Correspondence:}

Xueping Han

Department of Anesthesiology

The First Affiliated Hospital of Zhengzhou University

Institute of Clinical Medical Research of Henan

Universities

No. 1 Jianshe East Road,

Zhengzhou 450052, China

Phone: +86 37165662780

Fax: +8637165662780

XuepingHandoc@163.com

Received: Mar 23, 2017

Review: May 25, 2017

Accepted: June 26, 2017
Conflict of interest: none

Financial source: none

\begin{abstract}
${ }^{1}$ Research performed at Department of Anesthesiology, The First Affiliated Hospital, Zhengzhou University, Institute of Clinical Medical Research of Henan Universities, Zhengzhou, China.
\end{abstract}

